\title{
Immunogenicity of cell death driven by immune effectors
}

\author{
Lorenzo Galluzzi, ${ }^{1,2,3,4,5}$ Giulia Petroni, ${ }^{1}$ Guido Kroemer $^{5,6,7,8,9,10}$
}

To cite: Galluzzi L, Petroni G, Kroemer G. Immunogenicity of cell death driven by immune effectors. Journal for ImmunoTherapy of Cancer 2020;8:e000802. doi:10.1136/ jitc-2020-000802

LG and GK are joint senior authors.

Accepted 21 March 2020

\section{Check for updates}

(c) Author(s) (or their employer(s)) 2020. Re-use permitted under CC BY-NC. No commercial re-use. See rights and permissions. Published by BMJ.

For numbered affiliations see end of article.

\section{Correspondence to} Dr Lorenzo Galluzzi; deadoc80@gmail.com

Guido Kroemer; kroemer@orange.fr

\begin{abstract}
Whether cell death caused by T lymphocytes and natural killer (NK) cells would be immunogenic per se has been a matter of intense debate. Two back-to-back papers from the Melero's and Pardo's groups have now resolved this conundrum, demonstrating that $\mathrm{T}$ and NK cell-mediated cytotoxicity represents indeed a bona fide variant of immunogenic cell death.
\end{abstract}

Preclinical and clinical data accumulating over the past two decades demonstrate that-irrespective of treatment modalitythe success of cancer therapy most often relies on the activation of a robust immune response with systemic outreach. ${ }^{1}$ A key role in this context is played by so-called "epitope spreading," that is, the diversification of an adaptive immune response that initially targets only one or a few antigenic determinants but ultimately involves multiple T-cell clones specific for a variety of epitopes. Indeed, the vast majority of solid neoplasms display an elevated antigenic heterogeneity that generally compromises the efficacy of immune responses targeting a single antigen, as demonstrated by a plethora of therapeutic vaccines ultimately failing clinical testing. ${ }^{2}$ From an immunological perspective, epitope spreading obligatorily relies on several steps: (1) the release of novel antigenic material by cancer cells succumbing to the initial immune response; (2) the uptake of such material by antigen-presenting cells (APCs) and their migration to secondary lymphoid organs or tertiary lymphoid structures; and (3) the cross-priming of naive T-cell clones in the context of sufficient co-stimulatory signals for them to acquire effector functions. ${ }^{3}$ Thus, the death of cancer cells attacked by $\mathrm{CD}^{+}$ cytotoxic T lymphocytes (CTLs) must be able, at least in some instances, to re-initiate the cancer-immunity cycle and ultimately lead to the generation of additional CTLs with broadened specificity. ${ }^{3}$ Until now, however, formal demonstration that cellular cytotoxicity as mediated by CTLs and natural killer (NK) cells constitutes a bona fide variant of immunogenic cell death (ICD) ${ }^{4}$ was missing. Novel findings from the laboratories of $\mathrm{Dr}$ Melero and Dr Pardo elegantly resolve this conundrum as they demonstrate that cancer cells succumbing to immune effectors display sufficient antigenicity and adjuvanticity to initiate adaptive immune responses in immunocompetent hosts. ${ }^{56}$

By harnessing both parental and genetically modified mouse cancer cell lines including MC38 colorectal carcinoma cells and EL4 lymphoma cells, these authors elegantly demonstrated that cancer cells succumbing to antigen-specific CTLs or NK cells in vitro display surrogate markers of ICD such as the exposure of calreticulin (CALR) on the surface of the plasma membrane and the release of high mobility group Box 1 (HGMB1) into the extracellular space. When inoculated in immunocompetent syngeneic mice as a prophylactic vaccine, such dying cells conferred robust protection against a subsequent challenge with living cells of the same parental type, as well as with different cells genetically engineered to express a shared antigen. In particular, ovalbumin (OVA)-expressing MC38 exogenously pulsed with an immunogenic epitope from premelanosome protein (PMEL, best known as gp100) and then exposed to gp100-specific CTLs could successfully vaccinate C57BL/6 mice against a challenge with OVA-expressing EL4 cells (commonly known as EG7 cells). This elegant experimental design demonstrated epitope spreading in vivo. ${ }^{56}$

In line with an expanding literature on the molecular determinants of ICD, ${ }^{7}$ mouse cancer cells killed by CTLs or NK cells failed to protect syngeneic immunocompetent hosts from a challenge with antigenically compatible cells on the antibody-mediated depletion of $\mathrm{CD}^{+}$CTLs as well as on the whole-body knockout of Prf1, which encode the central cytotoxic molecule perforin 1. The prophylactic effect of cancer cells succumbing to CTLs or NK cells was also lost in mice lacking the HMGB1 receptor Tolllike receptor 4 (TLR4), as well as in mice 


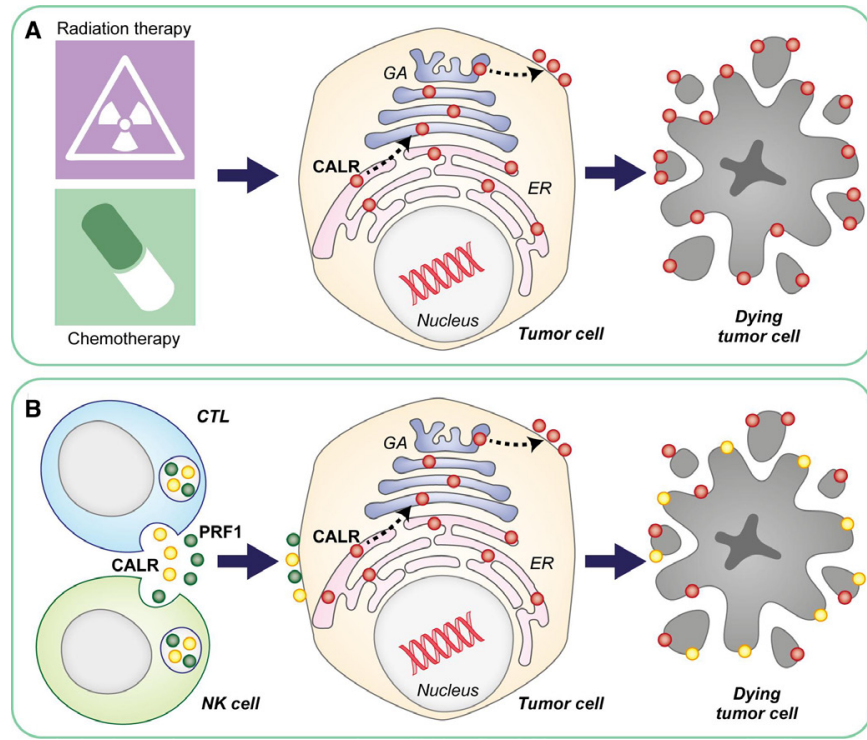

Figure 1 Cellular cytotoxicity as a potential source of adjuvanticity in trans. In the context of chemotherapyinduced and radiation therapy-induced immunogenic cell death (ICD), the phagocytosis-stimulatory molecule calreticulin (CALR) is exposed on the surface of dying cells as a consequence of anterograde transport from the endoplasmic reticulum (ER) to the plasma membrane via the Golgi apparatus (GA) (A). When ICD is initiated by cytotoxic T lymphocytes (CTLs) or natural killer (NK) cells, however, surface-exposed CALR may be provided by immune effectors (at least in part). This mechanism could be harnessed to improve the therapeutic efficacy of CAR T cells by maximizing their ability to deliver adjuvant-like signals to their targets, ultimately resulting in superior epitope spreading (B). PRF1, perforin 1.

that are deficient in cross-priming as a consequence of stimulator of interferon response cGAMP interactor 1 (Sting1) or basic leucine zipper transcription factor, ATF-like 3 (Batf3) deletion. ${ }^{5}$ Conversely, the results from Pardo and colleagues appear to challenge the current literature in proposing that caspase 3 (CASP3), a key regulator of apoptosis with multipronged immunosuppressive effects including the ability to shut down type I interferon production by STING $1,{ }^{8-10}$ is required for the immunogenicity of cancer cells succumbing to immune effectors. ${ }^{5}$ This interpretation was based on the ability of chemical pan-caspase inhibitor (Q-VD-OPh) and a dominant-negative variant of CASP3, but not the overexpression of BCL2-like 1 (BCL2L1, a potent antiapoptotic molecule best known as $\mathrm{BCL}_{\mathrm{L}}$ ), to reduce (to some degree) immunological protection conferred by cancer cells dying on attack by antigen-specific CTLs. However, Q-VD-OPh also blocks CASP8, which is essential for the immunogenicity of cell death because of its key function in ICD-associated CALR exposure. ${ }^{4}$ Moreover, the authors did not take into account the highly reduced sensitivity of CASP3-incompetent cells to death induced by CTLs or NK cells, which is expected to limit considerably the availability of antigenic material available for uptake by APCs and cross-priming. In line with this notion, BCL-X $\mathrm{L}_{\mathrm{L}}$ overexpression had little effects on sensitivity to death by immune effectors, which correlated with a minimal influence on vaccination efficacy. ${ }^{5}$ Thus, the impact of CASP3 on the efficacy of vaccination with cancer cells succumbing to CTLs and NK cells appears to be largely limited to its ability to precipitate cell death in this setting, which does not apply to ICD driven by other stimuli including radiation. ${ }^{8-10}$

Early work from the late Jurg Tschopp and colleagues demonstrated that the lytic granules of CTLs contain high levels of CALR, which was interpreted as a safeguard mechanism to prevent PRF1 activation by $\mathrm{Ca}^{2+}$ ions prior to granule exocytosis, largely relying on the ability of CALR to chelate $\mathrm{Ca}^{2+}$. ${ }^{11}$ Thus, it is possible that (at least part of) the CALR molecules detected on the surface of cancer cells attacked by CTLs (or NK cells) may be provided in trans by the latter (rather than in cis by the former) along with PRF1 and the other cytotoxic molecules contained in lytic granules. Vaccination experiments with $\mathrm{Calr}^{-1-}$ cancer cells are urgently awaited to clarify the relative contribution of endogenous vs exogenous CALR to the immunogenic potential of cellular cytotoxicity. Such a mechanistic exploration may be particularly important for the development of next-generation CAR T cells endowed with superior cytotoxic functions and also with the capacity to transfer adjuvanticity to their target, de facto favoring epitope spreading (figure 1). Irrespective of this and other unresolved conundrums, the current findings from Dr Melero's and Dr Pardo's groups shed new light onto the contribution of ICD to therapeutically relevant immune responses in the context of the cancerimmunity cycle.

\section{Author affiliations}

${ }^{1}$ Department of Radiation Oncology, Weill Cornell Medical College, New York, NY, USA

${ }^{2}$ Sandra and Edward Meyer Cancer Center, New York, NY, United States

${ }^{3}$ Caryl and Israel Englander Institute for Precision Medicine, New York, NY, United States

${ }^{4}$ Department of Dermatology, Yale School of Medicine, New Haven, CT, United States ${ }^{5}$ Université de Paris, Paris, France

${ }^{6}$ Equipe labellisée par la Ligue contre le cancer, INSERM U1138, Centre de Recherche des Cordeliers, Paris, France

${ }^{7}$ Metabolomics and Cell Biology Platforms, Gustave Roussy Comprehensive Cancer Institute, Villejuif, France

${ }^{8}$ Pôle de Biologie, Hôpital Européen Georges Pompidou, AP-HP, Paris, France ${ }^{9}$ Suzhou Institute for Systems Medicine, Chinese Academy of Sciences, Suzhou, China

${ }^{10}$ Department of Women's and Children's Health, Karolinska University Hospital, Stockholm, Sweden

Contributors $L G$ conceived the article and wrote the first version of the manuscript with input from GK. GP prepared the figure under supervision from LG. All authors approved the final version of the article.

Funding LG is supported by a Breakthrough Level 2 grant from the US Department of Defense (DoD), Breast Cancer Research Program (BRCP) (\#BC180476P1), by the 2019 Laura Ziskin Prize in Translational Research (\#ZP-6177, PI: Formenti) from the Stand Up to Cancer (SU2C), by a Mantle Cell Lymphoma Research Initiative (MCL-RI, PI: Chen-Kiang) grant from the Leukemia and Lymphoma Society (LLS), by a startup grant from the Dept. of Radiation Oncology at Weill Cornell Medicine (New York, US), by a Rapid Response Grant from the Functional Genomics Initiative (New York, US), by industrial collaborations with Lytix (Oslo, Norway) and Phosplatin (New 
York, US), and by donations from Phosplatin (New York, US), the Luke Heller TECPR2 Foundation (Boston, US) and Sotio a.s. (Prague, Czech Republic). GK is supported by the Ligue contre le Cancer (équipe labellisée); Agence National de la Recherche (ANR) - Projets blancs; ANR under the frame of E-Rare-2, the ERA-Net for Research on Rare Diseases; Association pour la recherche sur le cancer (ARC); Cancéropôle lle-de-France; Chancelerie des universités de Paris (Legs Poix), Fondation pour la Recherche Médicale (FRM); a donation by Elior; European Research Area Network on Cardiovascular Diseases (ERA-CVD, MINOTAUR); Gustave Roussy Odyssea, the European Union Horizon 2020 Project Oncobiome; Fondation Carrefour; High-end Foreign Expert Program in China (GDW20171100085 and GDW20181100051), Institut National du Cancer (INCa); Inserm (HTE); Institut Universitaire de France; LeDucq Foundation; the LabEx Immuno-Oncology; the RHU Torino Lumière; the Seerave Foundation; the SIRIC Stratified Oncology Cell DNA Repair and Tumor Immune Elimination (SOCRATE); and the SIRIC Cancer Research and Personalized Medicine (CARPEM).

Competing interests GK has been holding research contracts with Bayer Healthcare, Genentech, GlaxoSmithKline, Institut Mérieux, Kaleido, Lytix Pharma, Nucana, Oncolinx, PharmaMar, Samsara, Sotio and Vasculox. GK is on the Board of Directors of the Bristol Myers Squibb Foundation France. GK is a scientific co-founder of everlmmune, Samsara Therapeutics and Therafast Bio. LG received consulting fees from OmniSEQ, Astra Zeneca, Inzen and the Luke Heller TECPR2 Foundation, and he is member of the Scientific Advisory Committee of Boehringer Ingelheim, The Longevity Labs and OmniSEQ.

Patient consent for publication Not required.

Provenance and peer review Commissioned; externally peer reviewed.

Open access This is an open access article distributed in accordance with the Creative Commons Attribution Non Commercial (CC BY-NC 4.0) license, which permits others to distribute, remix, adapt, build upon this work noncommercially, and license their derivative works on different terms, provided the original work is properly cited, appropriate credit is given, any changes made indicated, and the use is non-commercial. See http://creativecommons.org/ licenses/by-nc/4.0/.

\section{REFERENCES}

1 Galluzzi L, Chan TA, Kroemer G, et al. The hallmarks of successful anticancer immunotherapy. Sci Trans/ Med 2018;10:eaat7807.

2 Vitale I, Sistigu A, Manic G, et al. Mutational and antigenic landscape in tumor progression and cancer immunotherapy. Trends Cell Biol 2019;29:396-416.

3 Chen DS, Mellman I. Elements of cancer immunity and the cancerimmune set point. Nature 2017;541:321-30.

4 Galluzzi L, Buqué A, Kepp O, et al. Immunogenic cell death in cancer and infectious disease. Nat Rev Immunol 2017;17:97-111.

5 Jaime-Sanchez Pet al. Cell death induced by cytotoxic CD8+ T cells is immunogenic and primes caspase- 3 dependent spread immunity against endogenous tumor antigens. J Immunother Cancer 2020.

6 Minute Let al. Cellular cytotoxicity is a form of immunogenic cell death. J Immunother Cancer 2020.

7 Galluzzi Let al. Consensus guidelines for the definition, detection and interpretation of immunogenic cell death. J Immunother Cancer 2020.

8 Rodriguez-Ruiz ME, Buqué A, Hensler M, et al. Apoptotic caspases inhibit abscopal responses to radiation and identify a new prognostic biomarker for breast cancer patients. Oncoimmunology 2019;8:e1655964.

9 Rongvaux A, Jackson R, Harman CCD, et al. Apoptotic caspases prevent the induction of type I interferons by mitochondrial DNA. Cell 2014;159:1563-77.

10 White MJ, McArthur K, Metcalf D, et al. Apoptotic caspases suppress mtDNA-induced STING-mediated type I IFN production. Cell 2014;159:1549-62.

11 Dupuis M, Schaerer E, Krause $\mathrm{KH}$, et al. The calcium-binding protein calreticulin is a major constituent of lytic granules in cytolytic T lymphocytes. J Exp Med 1993;177:1-7 